\title{
Considerations and Challenges with Characterizing Si/SiGe Interfaces
}

Ondrej Dyck ${ }^{1}$, Donovan Leonard ${ }^{1}$, Jonathan Poplawsky ${ }^{1}$, Emily Pritchett ${ }^{2}$, Andrey A. Kiselev ${ }^{2}$, Clayton A. Jackson ${ }^{2}$, and Lisa F. Edge ${ }^{2}$

${ }^{1}$ Center for Nanophase Materials Sciences, Oak Ridge National Lab, Oak Ridge, TN, USA

${ }^{2}$ HRL Laboratories, LLC., Malibu, CA, USA

Accurate and reproducible interface width measurements are integral in the refinement process for growth optimization of Si/SiGe heterostructures. However, in the Si/SiGe system, there is a lack of welldefined interfaces on the atomic length scale due to both the random nature of the SiGe alloy and to the fact that the SiGe is grown on one of the atomic species (Si) of which is it composed.

An established technique for characterizing interfacial widths through scanning transmission electron microscopy (STEM) is fitting the intensity profile with a sigmoid curve [1, 2] and taking values that are $90 \%$ and $10 \%$ of the asymptotic limits as the interface width (or some variation thereof). Using this technique, we find that for very thin $(<20 \mathrm{~nm})$ samples, the measured interface width can become erratic due to the random Ge concentration fluctuations within the SiGe. Fig. 1 illustrates the observed intensity fluctuations in the $\mathrm{Si}_{0.7} \mathrm{Ge}_{0.3}$ alloy in a thick (a) and thin (b) area of the cross-section sample (high angle annular dark field (HAADF) STEM images). This poses a challenge for simple sigmoid fitting as the interface "width" may fluctuate depending on localized Ge concentrations, even for an atomically sharp interface. Fig. 2(a) and 2(b) illustrate this further. A $\mathrm{Si}_{0.7} \mathrm{Ge}_{0.3}$ crystal was generated such that each atom had a $30 \%$ probability of being Ge, Fig. 2(a). Then, from the (100) surface of the crystal, Fig. 2(b) shows the surface where the first Ge atom is observed. When grown on Si, the Si atoms within the SiGe are indistinguishable from the substrate and a "rough" interface may be observed.

Figures 2(c)-(e) summarize a series of multislice HAADF-STEM simulations for perfect interfaces performed using the simulation software Dr. Probe [3]. While we observe some thickness dependence in Fig. 2(e), the spread in the data from one structure to the next confirms the difficulty with using a sigmoid fit for representing interface width. In particular, Figs. 2(c) and (d) highlight the cause of the conspicuous jump in interface width observed in one of the data series. Si and Ge is randomly added to the model, with respect to composition, to increase the model sample thickness. This causes unexpected fluctuations in the profile (arrows) and thus, erratic behavior in the sigmoid fit, which almost doubles the measurement.

The straightforward sigmoid fitting method works well for thicker samples where the average Ge concentration is represented in each atomic column. However, for thicker samples, image resolution is reduced and chemical information is obscured by neighboring columns due to cross-talk [4]. This presents a problem for reliably resolving atomically perfect interfaces. New analysis methods are needed to define interface width on thin samples in a straightforward manner to facilitate the high sample throughput required for growth optimization.

\section{References:}

[1] E. Luna et al., Appl. Phys. Lett. 92, p. 141913 (2008).

[2] E. Luna et al., Appl. Phys. Lett. 96, p. 021904 (2010).

[3] J. Barthel, http://www.er-c.org/barthel/drprobe/ (2015). 
[4] P. M. Voyles et al., Ultramicroscopy 96, p. 251 (2002).

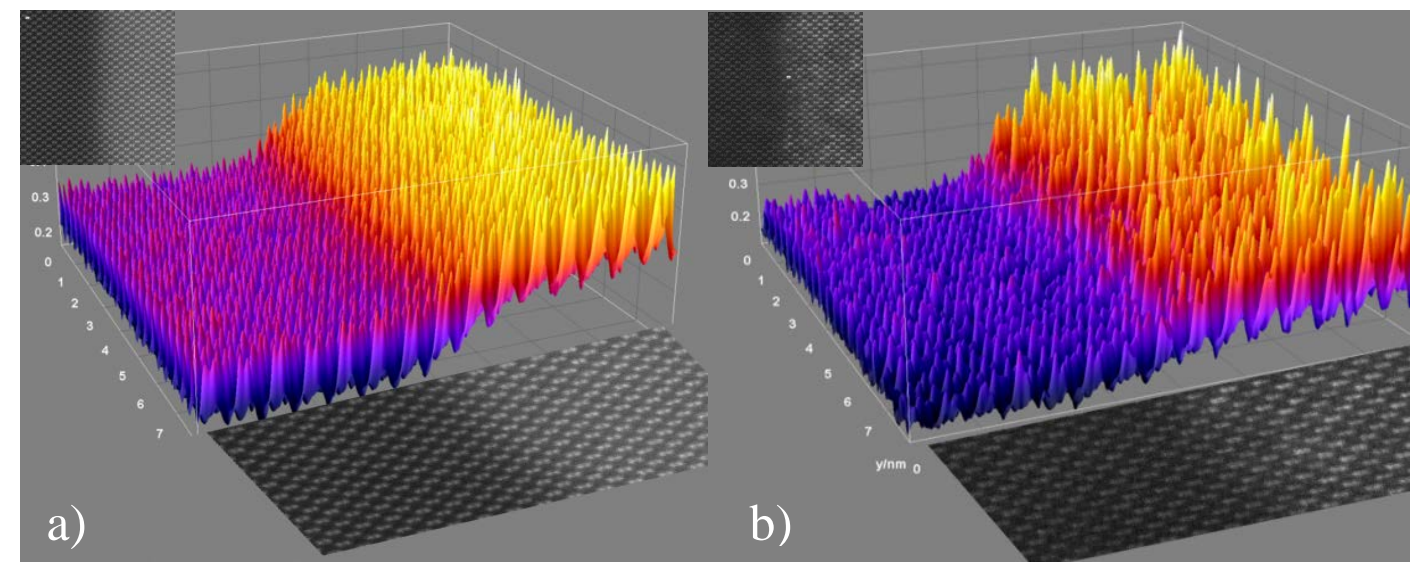

Figure 1. Intensity profiles across the Si/SiGe interface for thick (a) and thin (b) areas of the same sample. Original images are inset and representative portions are displayed underneath the intensity profiles. A marked increase in the relative intensity variations in the SiGe is due to the randomly occurring Ge occupation in the material. The intensity profile surfaces were smoothed to reduce noise.

a)
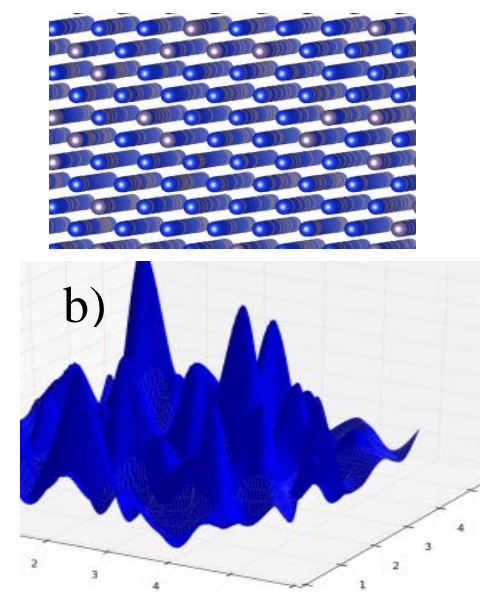
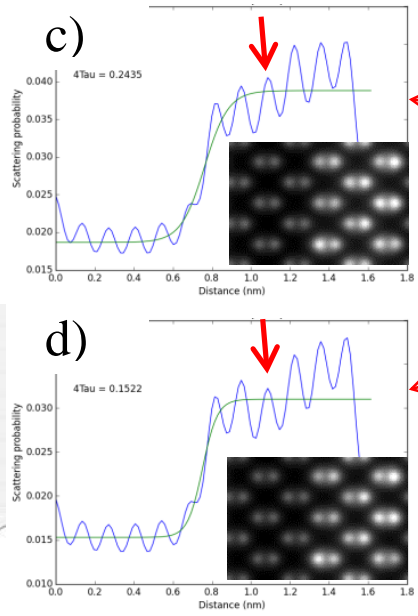

e) Multislice Simulated Interface Widths

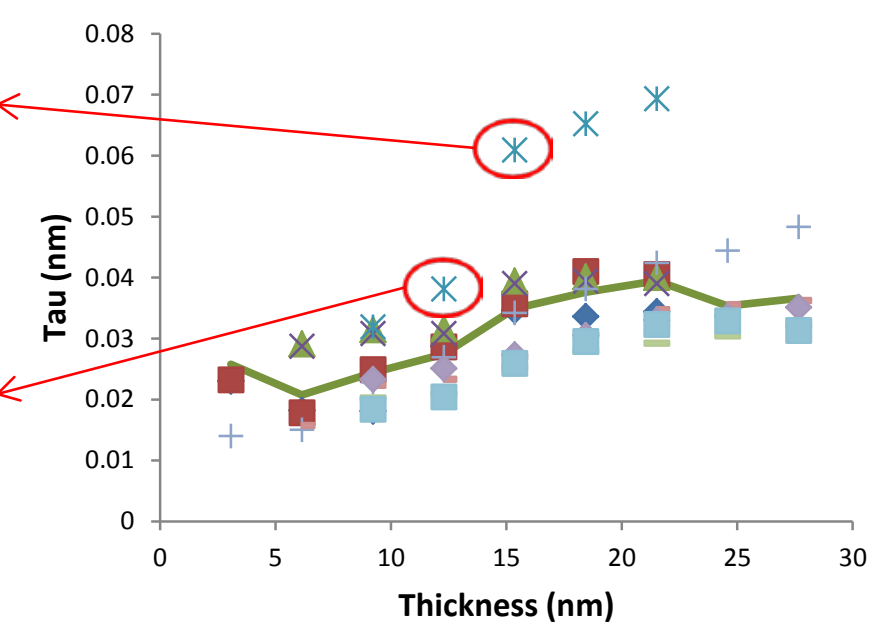

Figure 2. (a) A simulated $\mathrm{Si}_{0.7} \mathrm{Ge}_{0.3}$ crystal viewed just off the 100 zone axis. Blue=Si; grey=Ge. Each atom has a 30\% chance of being Ge. (b) Obtained from an analysis of (a) representing the distance to the first observed Ge atom in each column. In an ordered alloy this surface would vary by no more than half a unit cell distance, but in a random alloy fluctuations of several nanometers (units are in $\mathrm{nm}$ ) are observed. This surface represents a possible configuration of an atomically sharp interface with Si and highlights the challenge for distinguishing a perfect interface. (c) and (d) Line profiles fit with sigmoid curves of simulated HAADF-STEM images of the $\mathrm{Si} / \mathrm{Si}_{0.7} \mathrm{Ge}_{0.3}$ interface boundary (simulated images inset). Observed small changes in intensity (arrows) that are randomly located can cause large changes in the sigmoid fit. (e) A plot of interface width measurements as a function of sample thickness. We observe a trend of increasing interface width with thickness but the spread in values associated with random fluctuations in Ge concentration is large, indicating that precise measurements are not possible on thin samples using this technique. 